\title{
Cultural differences in music features across Taiwanese, Japanese, and American markets
}

\author{
Kongmeng Liew ${ }^{\text {Corresp., } 1,2}$, Yukiko Uchida ${ }^{3}$, Igor de Almeida ${ }^{4}$ \\ ${ }^{1}$ Graduate School of Science and Technology, Nara Institute of Science and Technology, Ikoma City, Nara Prefecture, Japan \\ 2 Graduate School of Human and Environmental Studies, Kyoto University, Kyoto City, Kyoto Prefecture, Japan \\ 3 Kokoro Research Center, Kyoto University, Kyoto City, Kyoto Prefecture, Japan \\ 4 Institute of Liberal Arts, Otemon Gakuin University, Ibaraki City, Osaka Prefecture, Japan \\ Corresponding Author: Kongmeng Liew \\ Email address: liew.kongmeng@is.naist.jp
}

Background. Preferences for music can be represented through music features. The widespread prevalence of music streaming has allowed for music feature information to be consolidated by service providers like Spotify. In this paper, we demonstrate that machine learning classification on cultural market membership (Taiwanese, Japanese, American) by music features reveals variations in popular music across these markets.

Methods. We present an exploratory analysis of 1.08 million songs centred on Taiwanese, Japanese and American markets. We use both multiclass classification models (Gradient Boosted Decision Trees (GBDT) and Multilayer Perceptron (MLP)), and binary classification models, and interpret their results using variable importance measures and Partial Dependence Plots. To ensure the reliability of our interpretations, we conducted a follow-up study comparing Top-50 playlists from Taiwan, Japan, and the US on identified variables of importance.

Results. The multiclass models achieved moderate classification accuracy (GBDT $=0.69, \mathrm{MLP}=0.66)$. Accuracy scores for binary classification models ranged between 0.71 to 0.81 . Model interpretation revealed music features of greatest importance: Overall, popular music in Taiwan was characterised by high acousticness, American music was characterized high speechiness, and Japanese music was characterized by high energy features. A follow-up study using Top-50 charts found similarly significant differences between cultures for these three features.

Conclusion. We demonstrate that machine learning can reveal both the magnitude of differences in music preference across Taiwanese, Japanese, and American markets, and where these preferences are different. While this paper is limited to Spotify data, it underscores the potential contribution of machine learning in exploratory approaches to research on cultural differences. 


\section{Cultural Differences in Music Features Across Taiwanese,} 2 Japanese, and American Markets

3

4 Kongmeng Liew ${ }^{1,2}$, Yukiko Uchida ${ }^{3}$, Igor de Almeida ${ }^{4}$

5

$6{ }^{1}$ Graduate School of Science and Technology, Nara Institute of Science and Technology, Ikoma,

7 Nara Prefecture, Japan

$8{ }^{2}$ Graduate School of Human and Environmental Studies, Kyoto University, Kyoto, Kyoto

9 Prefecture, Japan

$10{ }^{3}$ Kokoro Research Center, Kyoto University, Kyoto, Kyoto Prefecture, Japan

$11{ }^{4}$ Institute of Liberal Arts, Otemon Gakuin University, Ibaraki, Osaka Prefecture, Japan 12

13 Corresponding Author:

14 Kongmeng Liew ${ }^{1}$

15 8916-5 Takayama-cho, Ikoma, Nara, 630-0192, Japan

16 Email address: liew.kongmeng@is.naist.jp 


\section{Abstract}

18

Background. Preferences for music can be represented through music features. The widespread prevalence of music streaming has allowed for music feature information to be consolidated by service providers like Spotify. In this paper, we demonstrate that machine learning classification on cultural market membership (Taiwanese, Japanese, American) by music features reveals variations in popular music across these markets.

Methods. We present an exploratory analysis of 1.08 million songs centred on Taiwanese, Japanese and American markets. We use both multiclass classification models (Gradient Boosted Decision Trees (GBDT) and Multilayer Perceptron (MLP)), and binary classification models, and interpret their results using variable importance measures and Partial Dependence Plots. To ensure the reliability of our interpretations, we conducted a follow-up study comparing Top-50 playlists from Taiwan, Japan, and the US on identified variables of importance.

Results. The multiclass models achieved moderate classification accuracy (GBDT $=0.69$, MLP $=0.66)$. Accuracy scores for binary classification models ranged between 0.71 to 0.81 . Model interpretation revealed music features of greatest importance: Overall, popular music in Taiwan was characterised by high acousticness, American music was characterized high speechiness, and Japanese music was characterized by high energy features. A follow-up study using Top-50 charts found similarly significant differences between cultures for these three features.

Conclusion. We demonstrate that machine learning can reveal both the magnitude of differences in music preference across Taiwanese, Japanese, and American markets, and where these 
41

42

43

44

45

46

47

48

49

50

51

52

53

54

55

56

57

58

59

60

61

62

63

64

65

66

67

68

69

70

71

72

73

74

75

76

77

78

79

preferences are different. While this paper is limited to Spotify data, it underscores the potential

contribution of machine learning in exploratory approaches to research on cultural differences.

\section{Introduction}

With 219 million active listeners a month, and a presence in over 60 countries, Spotify is one of the largest music streaming service providers in the world (as of 2020, Schwind et al., 2020). To facilitate such a service, they maintain a database of music features for all songs in their service, that is made publicly accessible through the Spotify API (Application Programme Interface). This database contains a wealth of meta and music feature data, that researchers have been using to research human behaviour and engagement with music. For example, Park and colleagues (Park et al., 2019) analysed data from 1 million individuals across 51 countries and uncovered consistent patterns of music preferences across day-night cycles: relaxing music was more commonly played at night, and energetic music during the day. Pérez-Verdejo and colleagues (2020) found that popular hit songs in Mexico shared many similarities to global hit songs. Spotify's music features have also been used to examine songs in clinical and therapeutic settings, with Howlin and Rooney (2020) finding that songs used in previous pain management research, if chosen by the patient, tended to have high energy, danceability, and lower instrumentalness features, than experimenter-chosen songs.

In this paper, we use Spotify features to examine how cultures differ in music preferences, through a bottom-up, data-driven analysis of music features across cultures. Here, we quantify music preference through music features, following past research (e.g., Fricke et al., 2019). Music plays a huge role in human society, be it in emotion regulation or for social displays of identity and social bonding (Groarke \& Hogan, 2018; Dunbar, 2012). These are often embedded in the cultural norms and traditions of the listener. In other words, understanding how music differs across cultures may reflect corresponding cultural differences in the sociocultural context that shape our individual preferences towards certain types of music over others. Thus, understanding how music differs between these cultural markets, e.g., by examining their features, may then shed light on possible cultural differences, which can guide follow-up research by generating novel hypotheses, or in supporting various theories on cultural differences aside from music (see below).

To achieve this aim, we rely on machine learning classification of songs based on cultural markets (i.e., culture of origin), as interpretation of these models may reveal insight into how (and which) features differ between cultures. We utilize data seeded originally on Taiwanese, American and Japanese Top-50 lists. This arguably aligns more towards a sociolingual distinction of cultural membership, and not a country-based sampling commonly used in culture research. Our reasoning was that this more closely resembles the differentiated cultural markets that artists of a certain language operate in, that often transcend country boundaries. For example, popular Chinese music meant for a Chinese cultural market often subsumes artists from wider Chinese cultural origins (in countries and territories like Taiwan, Hong Kong, Singapore, 
80

81

82

83

84

85

86

87

88

89

90

91

92

93

94

95

96

97

98

99

100

101

102

103

104

105

106

107

108

109

110

111

112

113

114

115

116

117

118

119

and Malaysia; Fung, 2013; Moskowitz, 2009). As such, in this paper, these were considered to belong to a 'Chinese' cultural market (including music from language subtypes and dialects). Japanese music was similarly treated as belonging to a 'Japanese' market, and music from Western (Anglo-European) cultural origins (e.g., the US, UK, Canada, and Australia) were considered as belonging to a 'Western' cultural market.

Past approaches towards music preference through Spotify have largely focused on curated lists of Top-50 or Top-200 popular songs (e.g., Pérez-Verdejo et al., 2020; Febirautami, Surjandari \& Laoh, 2019). While such lists are often diluted by the inclusion of 'global' hit songs, they nevertheless provide a window to examine culturally based music preferences. Accordingly, we also conduct a follow-up study using these Top-50 lists from Taiwan, Japan, and the US to ensure the reliability of our interpretations (from the classification model).

\section{Cultural differences in music preference}

Typically, most of the research in cultural differences in the psychological literature has come from top-down, theoretical approaches. These have been instrumental in shaping the field, by increasing awareness of systematic ways by which people from cultures are different. One of the most instrumental differences is in the independence and interdependence of the self (Markus \& Kitayama, 1991; 2010). Westerners generally tend to be independent, in that they prioritise the autonomy and uniqueness of internal (self) attributes. In contrast, East Asians generally tend to be interdependent, where their concept of self is intricately linked to close social relationships. This has been shown to have implications on music preferences through differences in desirability of emotions. For example, Westerners generally tend to view happiness as a positive and internal hedonic experience to be maximised where possible (Joshanloo \& Weijers, 2014). As such, Western music preference tends towards high-arousal music, possibly in the search of strong 'happiness' experiences. East Asians, however, view happiness as a positive feeling associated with harmonious social relationships, in contrast to the hedonic, high-arousal definition in Western contexts (citation). Consistently, East Asian preferences for music do not have this high-arousal component, and is more calm, subdued, and relaxing (Tsai, 2007; Uchida \& Kitayama, 2009; Park et al., 2019).

However, such theoretically based analyses may overrepresent Western cultures in research and literature. Consequently, cultural differences within similar, non-Westernised spheres are not well understood, due to the lack of pre-existing theory. For example, Chinese and Japanese cultures are often grouped together in cross-cultural research as a representation of East Asian collectivism, that functions as a comparative antithesis to Western findings (e.g., Heine \& Hamamura, 2007). Yet, research has also uncovered differences between China and Japan that cannot be explained by these theories (Muthukrishna et al., 2020). As such, cultural differences within East Asia are not well understood in the psychological literature, and few theories exist to offer predictions on differences in music preference between these cultures.

Our solution was to examine music as cultural products from the bottom-up. Doing so would reduce the effect of experimenter bias in guiding theory formation and interpretation, 
120

121

122

123

124

125

126

127

128

129

130

131

132

133

134

135

136

137

138

139

140

141

142

143

144

145

146

147

148

149

150

151

152

153

154

155

156

157

158

159

when examining a wide database of music features. Cultural products are behavioural manifestations of culture that embody the shared values and collective aesthetics of a society (Morling \& Lamoreaux, 2008; Lamoreaux \& Morling, 2012; Smith et al., 2013). This implies that music consumption behaviour underscores culturally based attitudes, cognitions and emotions that afford preferences for certain congruent types of music. For example, in a crosscultural comparison between Brazil and Japan, de Almeida and Uchida (2018) found that Brazilian song lyrics contained higher frequencies of positive emotion words and lower frequencies of neutral words than Japanese lyrics. This was consistent and reflective of their respective cultural emphases on emotion expressions (see Triandis et al., 1984; Uchida \& Kitayama, 2009), and showed that comparing music 'products' elucidated differences between the collective shared values of different cultures. Past research on cultural products have relied on both popularity lists (charts; e.g., Askin \& Mauskapf, 2017), and on artifacts produced by a culture (e.g., Tweets: Golder \& Macy, 2011; newspaper articles: Bardi, Calogero, \& Mullen, 2008). We utilize both methods, and propose that examining cultural differences in 'music' products on a large-scale may provide potential insight into the sociocultural circumstances that give rise to these differences.

\section{The Present Research}

We adopted a data-driven, bottom-up approach to explore music preferences between cultures/industries through musical features for this study through machine learning. I.e., we first train a multiclass model to classify songs as belonging to (originating from) Chinese (Taiwanese), Japanese, or Western (American) markets. This is to establish the presence and magnitude of discernible cultural differences in music features. Next, we decompose the model by training 3 binary machine learning classifiers to classify songs as belonging to one culture or another. By applying model interpretation techniques on these models (such as Partial Dependence Plots [PDPs]), we aim to discover the specific difference in preferred musical features between Chinese (Taiwanese)-Japanese markets, Chinese (Taiwanese)-Western (American) markets, and Japanese-Western (American) markets. We aimed to include as many songs as possible that were produced from these respective culture-based music industries to observe systematic trends and differences from as wide a range of musical styles and genres within these industries as possible. Finally, we examine the generalizability of these interpretations by conducting a follow-up study on Top-50 songs from Taiwan, Japan, and the US. If the identified features of difference present in songs produced by a cultural market were indeed representative of cultural differences in music preferences, we expect that these features should also show consistent differences for their respective Top-50 (popular) songs.

\section{Materials \& Methods}

\section{Overview}

To explore differences between cultures, we used machine learning to classify a database of Chinese, Japanese, and English songs into their respective cultural (linguistic) markets. As this 
160

161

162

163

164

165

166

167

168

169

170

171

172

173

174

175

176

177

178

179

180

181

182

183

184

185

186

187

188

189

190

191

192

193

194

195

196

197

198

199

was a multiclass classification problem, we conducted the analysis twice using gradient boosted decision trees (GBDTs) and artificial neural networks (multi-layer perceptron, MLP) that are inherently capable of multiclass classification. This was also to examine the consistency in results between two differing methods of analysis, and strengthen the reliability of the analyses.

To infer the features that accounted for cultural differences, we use model interpretation techniques, namely relative feature importance (RFI; Friedman, 2001), permutational feature importance (PFI; Fisher, Rudin, \& Dominici, 2018), and partial dependence plots (PDPs, Friedman, 2001), to examine and visualise the relationships between the feature and its influence on the probability of classification.

\section{Data mining}

We accessed the Spotify Application Programme Interface (API) through the 'spotifyr' wrapper (Thompson, Parry, \& Wolff, 2019) in R, to obtain song-level music feature information from Chinese, Japanese and English artists from the Spotify database. This was through a pseudo-snowball sampling method: we relied on Spotify's recommendation systems (the 'get_related_artists' function) to recommend artists related to those in the official Spotify Top-50 chart playlists for Taiwan, Japan, and the US respectively and created a list of artists per country. We then used the same method to obtain another list of recommended artists to these respective 'lists', for up to 6 iterations, in order to obtain comparable sample sizes between these three markets. We also excluded all non-Chinese, non-Japanese, and non-English (language) artists from the respective list. This was through an examination of the associated genres for each artist, which often contained hints to their cultural origins (e.g., J-pop, J-rock, Mandopop). Artists that did not have listed genres were checked manually by the researchers. This resulted in a final $\mathrm{N}($ artists $)=10259($ Japanese $=2587$; English $=2466$; Chinese $=5206)$. All song-level feature information for all artists were then obtained from the Spotify database. Duplicates (such as the same song being rereleased in compilation albums) were removed, for a total of $\mathrm{N}$ (songs) = $1810210($ Japanese $=646440$; Chinese $=360101$; English $=803669)$. To ensure class balances, we randomly downsampled the Japanese and English samples to match the Chinese sample, resulting in a final $\mathrm{N}($ songs $)=1080303$.

\section{Data handling and analysis}

Except for 'key' and 'time signature', all Spotify features were inputted as features in the classification models. These were: 'danceability', 'energy', 'loudness', 'speechiness', 'acousticness', ‘instrumentalness', 'liveness', 'valence', 'tempo', 'duration' (ms), and 'mode'. A list of definitions for these features is available in Table 1. These were to classify songs according to their cultural membership (Chinese, English, or Japanese), as the outcome variable. The data was split into a training and testing set along a 3:1 ratio. Parameters for the GBDT model and weights for the MLP model were tuned through 5-fold cross validation on the training set. We also examined RFI scores for each model. For GBDT, this was a measure of the proportion that a feature was selected for stratification in each iterative tree, and for MLP, this 
200

201

202

203

204

205

206

207

208

209

210

211

212

213

214

215

216

217

218

219

220

221

222

223

224

225

226

227

228

229

230

231

232

233

234

235

236

237

238

239

was based on PFI, which measures the resultant error of a model when each feature is iteratively shuffled - the greater the error, the larger the influence a feature exerts on the outcome variable (Fisher, Rudin, \& Dominici, 2018; Molnar, 2019). We then simplified the classification problem by splitting it into 3 separate binary classifications: Japanese-Chinese, Japanese-English, and Chinese-English. GBDTs and MLP models were conducted for these three comparisons, and in addition to RFI measures, we visualised the effect of each variable using PDPs. These show the averaged marginal effect of a feature on the outcome variable in a machine learning model, and is useful to glean an understanding of the nature of the relationship between these variables. PDPs were conducted through the 'pdp' package (Greenwell, 2017), and PFIs were conducted through the 'iml' package (Molnar, Bischl, \& Casalicchi, 2019). Machine learning was conducted through the 'gbm' package Greenwell et al., 2019) for GBDTs, and the 'nnet' package (Venables \& Ripley, 2002) for MLPs, via the 'caret' wrapper (Kuhn, 2019) in R (R Core Team, 2019). All R scripts used for data mining and analysis are available in our OSF repository (Note to reviewers: anonymous review link:

https://osf.io/d3cky/?view only=cc89c024cedb4bfd8401544032e505a1).

\section{Results}

\section{Descriptives}

Table 2 reports the descriptive medians, lower/upper quantiles, and missing data for each feature per culture. The full list of artists, genres, and songs are available in our OSF repository (Note to reviewers: anonymous review link:

https://osf.io/d3cky/?view only=cc89c024cedb4bfd8401544032e505al). Additionally, we note that while our database of songs spans as early as the 1950s, most of the songs in our database were from the mid-2000s to 2020 (see Figure 1).

\section{Multiclass classification (Chinese-Japanese-English)}

For the GBDT model, the parameter tuning resulted in $\mathrm{N}($ trees $)=150$, interaction depth $=$ 3 , alongside default parameters of shrinkage $=0.1$, and number of minimum observations per node $=10$. The GBDT achieved a classification accuracy of $0.682,95 \% \mathrm{CI}(0.680,0.683)$, significantly above the no information rate (NIR) of $0.333, p<.0001$. Aside from the input and output layers, we used a MLP model consisting of 1 hidden layer with 5 nodes. The MLP model achieved a slightly lower accuracy score of $0.660,95 \% \mathrm{CI}(0.659,0.662)$, but was still

significantly above the NIR of $0.333, p<.0001$. RFIs for both models are reported in Table 3 .

\section{Binary classifications}

We first unpack the Chinese-Japanese model: For the GBDT model, the parameter tuning resulted in $\mathrm{N}$ (trees) $=150$, interaction depth $=3$, and no changes were made to the other default parameters (as above). The GBDT achieved a classification accuracy of $0.784,95 \% \mathrm{CI}(0.783$, $0.786), \mathrm{AUC}=0.865$, significantly above the no information rate (NIR) of $0.500, p<.0001$. The MLP model achieved a comparable accuracy score of $0.766,95 \% \mathrm{CI}(0.764,0.768), \mathrm{AUC}=$ 
2400.844 , significantly above the NIR of $0.500, p<.0001$. Next, the Chinese-English model: For the 241 GBDT model, the parameter tuning resulted in $\mathrm{N}($ trees $)=150$, interaction depth $=3$, and no 242 changes were made to the other default parameters. The GBDT achieved a classification 243 accuracy of $0.807,95 \% \mathrm{CI}(0.805,0.809)$, $\mathrm{AUC}=0.885$, significantly above the no information 244 rate (NIR) of $0.500, p<.0001$. The MLP model achieved a comparable accuracy score of 0.803 , $24595 \% \mathrm{CI}(0.801,0.805), \mathrm{AUC}=0.880$, significantly above the NIR of $0.500, p<.0001$. Finally, the 246 Japanese-English model: For the GBDT model, the parameter tuning resulted in $\mathrm{N}($ trees $)=150$, 247 interaction depth $=3$, and no changes were made to the other default parameters. The GBDT 248 achieved a classification accuracy of $0.713,95 \% \mathrm{CI}(0.711,0.715), \mathrm{AUC}=0.797$, significantly 249 above the no information rate (NIR) of $0.500, p<.0001$. The MLP model achieved a comparable 250 accuracy score of $0.709,95 \% \mathrm{CI}(0.707,0.711)$, AUC $=0.791$, significantly above the NIR of $2510.500, p<.0001$. All RFIs and PFIs are reported in Table 4. Additionally, the 2 most important

252

253

254

255

256

257

258

259

260

261

262

263

264

265

266

267

268

269

270

271

272

273

274

275

276

277

278

279

features are visualised by PDPs in Figure 2. A visual inspection of the PDPs suggests that English music is higher than both Japanese and Chinese music in speechiness, Chinese music is higher than both Japanese and English music in acousticness, and Japanese music is higher than English and Chinese music in energy. In comparing Japanese and Chinese music, we note that acousticness and energy were also present, but were identified only in the GBDT model. In contrast, the MLP model identified loudness and instrumentalness as higher in Japanese music than Chinese music.

Overall, this suggests that, unlike English-Japanese or English-Chinese comparisons which were markedly different on a few main features, the differences between Chinese and Japanese music were spread widely across the various features. Consequently, despite relying on different 'important variables', both the MLP and GBDT managed to achieve a comparably high classification accuracy, with the GBDT outperforming the MLP for all classification tasks (results from DeLong's tests are available on our OSF repository).

\section{Additional Analyses}

We also visualised the changes in features over time for speechiness, acousticness, energy, instrumentalness, and loudness, from 2000 to 2020. Feature information for songs before 2000 were excluded due to the markedly smaller sample. Other than instrumentalness, which showed a notable decrease over time in Japanese songs, the remaining four features showed stability over time. This suggests that the differences in preference highlighted by the RFIs, PFIs and PDPs could indicate long term cultural preferences for music.

\section{Follow-up Study}

We obtained a second round of data (approximately one year later) from Top-50 lists for Japan, Taiwan and the USA. Focusing on the identified features of speechiness, acousticness, energy, instrumentalness, and loudness from the previous study, Kruskal-Wallis tests revealed a significant effect of energy on speechiness $\left(\chi^{2}(2)=30.5, p<.001\right)$, acousticness $\left(\chi^{2}(2)=24.5, p\right.$ $<.001)$, energy $\left(\chi^{2}(2)=21.0, p<.001\right)$, and loudness $\left(\chi^{2}(2)=33.5, p<.001\right)$, but no significant 
280

281

282

283

284

285

286

287

288

289

290

291

292

293

294

295

296

297

298

299

300

301

302

303

304

305

306

307

308

309

310

311

312

313

314

315

316

317

318

319

effect was observed for instrumentalness $\left(\chi^{2}(2)=1.4, p=.49\right)$. For speechiness, post-hoc DwassSteel-Critchlow-Fligner pairwise comparisons revealed that USA was significantly higher than Taiwan (W=7.34, $p<.001)$ and Japan ( $\mathrm{W}=5.91, p<.001)$, but no significant difference was observed between Taiwan and Japan $(\mathrm{W}=-1.48, p=.55)$. For acousticness, Taiwan was significantly higher than Japan ( $\mathrm{W}=5.97, p<.001$ ) and the USA ( $\mathrm{W}=6.11, p<.001)$, but no significant difference was observed between the USA and Japan ( $\mathrm{W}=0.53, p=.93)$. For energy, Japan was significantly higher than Taiwan ( $\mathrm{W}=6.01, p<.001)$, and the USA (W $=4.35, p=$ .006), but no significant difference was observed between Taiwan and the USA (W $=2.88, p=$ .103). For loudness, Japan was significantly higher than Taiwan ( $\mathrm{W}=7.44, p<.001)$ and the USA ( $\mathrm{W}=6.36, p<.001$ ), but no significant difference was observed between Taiwan and the USA ( $\mathrm{W}=2.20, p=.27)$. Finally, for instrumentalness, no significant difference was observed between Japan and Taiwan ( $\mathrm{W}=0.61, p=.90)$, Japan and the USA ( $\mathrm{W}=1.67, p=.48)$, or Taiwan and the USA ( $\mathrm{W}=1.01, p=.75)$.

In short, with the exception of instrumentalness, Top-50 playlists obtained one year later nevertheless demonstrate strong consistency with the earlier results. American Top-50 songs are higher than both Japanese and Taiwanese Top-50 songs in speechiness, Taiwanese Top-50 songs are higher than both Japanese and American Top-50 songs in acousticness, and Japanese Top-50 songs are higher than American and Taiwanese music in energy and loudness. However, instrumentalness, that was originally identified as a variable of importance for the MLP model, did not consistently differ between cultures. Indeed, Figure 3 shows that instrumentalness in Chinese music is inconsistent, with strong fluctuations depending on year. More research is needed to determine if instrumentalness is indeed a preferred feature in Taiwanese markets or merely a passing trend.

\section{Discussion}

Across the multiclass and subsequent binary classification tasks, both the GBDT and MLP models were able to consistently classify songs by cultural market with moderately high accuracy, and the GBDT was often marginally better than the MLP for this purpose. This suggests that the patterns of difference between cultural markets were robust enough to be detected by two different algorithms. A comparison of accuracy scores suggested that the difference between Chinese and Japanese music afforded higher accuracy to the models, than Japanese and English differences. While this could be for several reasons, we speculate a possibility in that music preferences between Japanese and Chinese cultures differed greater that Japanese and English differences. Such a reasoning would support growing calls for decentralisation and internationalisation of psychological research (Heine \& Ruby, 2010; Heinrich, Heine, \& Norenzayan, 2010; Cheung, 2012), in showing that Japanese and Chinese speaking cultures, sometimes thought to be homogenous in cross-cultural research, may actually be more different that previously assumed.

A visual inspection of the PDPs and feature importance scores provide some indicator of where these differences lie. Apart from instrumentalness, all identified feature importance 
320

321

322

323

324

325

326

327

328

329

330

331

332

333

334

335

336

337

338

339

340

341

342

343

344

345

346

347

348

349

350

351

352

353

354

355

356

357

358

359

proved to be different across (geographical) cultures in similar directions in the follow-up study. This implies that cultural music preferences are reflected both in the music produced by a culture for their respective industry or market, as well as the overall music preferences by geographically bound members of that culture. While this paper does not empirically explore the underlying cultural mechanisms that may account for these differences, this is nevertheless a starting point for future research to continue from, and we speculate on some interpretations for these results. Western music similarly differed from Chinese and Japanese music through higher speechiness. One explanation could be prosodic bias, in that normal spoken Mandarin Chinese inherently contains more pitch movements than English (Hirst, 2013), and consequently, what may be perceived as 'speech-like' by Chinese listeners may not correspond to high speechiness scores. However, this can also be explained through previous research on emotion-arousal preferences in the Western and East-Asian contexts. The high speechiness score in English music could indicate larger preferences for hip-hop and rap music. Rap-music has seen a dramatic increase in popularity in Western markets from the 1980s (Mauch et al., 2015), and has been shown to express and embody high-arousal emotions like anger (e.g., Hakvoort, 2015), and its relative popularity in Anglo-American cultures could be representative on cultural preferences towards these high arousal emotions described earlier (Tsai, 2007), compared to Japanese and Chinese cultures.

One feature that differentiated Chinese from English and Japanese music was high acousticness. This points to lower use of electronic instruments in the production process, and may suggest a preference for more organic, natural sounds in Chinese music. Energy appeared to be more important in Japanese than English or Chinese music. We posit that energy preferences in Japan (defined by Spotify as a combination of loudness, complexity, timbre, dynamic range, and noise) could be due to remnants of traditional music aesthetics, that overlap considerably with energy definitions (e.g., beauty in noise/simplicity in complexity: sawari, wabi-sabi, see Deva, 1999; Anderson, 2014; Okuno, 2015). On the surface, this could be similarly concluded from increased loudness features in Japanese over Chinese music (from the follow-up study), but the U-shaped relationship between energy and Japanese/Chinese music classification seen in Figure 2 suggests a deeper nuance that requires further research.

Finally, we consider the strengths and limitations our exploratory approach. Comparing music features offer a greater insight into behavioural and consumption patterns of music preference across cultural spheres. In doing so, we uncover systematic differences between groups that, while being consistent with previous literature, also offer new insight into how cultures differ, that future research can build from in understanding societies. Unfortunately, we were unable to eliminate certain sample biases from our dataset: we assumed our Chinese data to be representative of Chinese music in general, but Spotify is not (as of 2021) active in China despite the inclusion of several mainland Chinese artists in the database. Instead, our findings represented Chinese-speaking listeners in Taiwan and Hong Kong, along with possibly Malaysia and Singapore, who may have differing values and preferences from mainland Chinese listeners, particularly given differences in demographics of users and variation in dialect. Moreover, using 
360

361

362

363

364

365

366

367

368

369

370

371

372

373

374

375

376

377

378

379

380

381

382

383

384

385

386

387

388

389

390

391

392

393

394

395

396

397

398

399

the Spotify API limited our selection of music features to those available in the API. Future studies could examine music features through publicly available software (e.g., MIRtoolbox; Lartillot, Toiviainen, \& Eerola, 2008) that have both greater amounts of features and more transparent documentation.

On the other hand, our strengths include our comparisons of features, as opposed to genre, that allowed for validity in comparing cultures because of universality in the perceptual properties of music (Savage et al., 2015). This enabled us to conclude that any differences in music features would be due to preference for those features. By contrast, comparing preferences by genre differences across cultures could have introduced confounds to the investigation, as genre is not homogenous across cultures (see Bennett, 1999).

\section{Conclusions}

In sum, we demonstrated the variability of music preferences across Chinese (Taiwan), Japanese, and Western (American) cultural markets, and identified the features that best account for these differences. In particular, Chinese music was marked by high acousticness, AngloAmerican Western music was marked by high speechiness, and Japanese music appeared to be marked by high energy. While we speculated on some reasons why this would be so, future research is needed to validate these theories to develop a holistic understanding of popular music preferences in Chinese and Japanese cultures. As music is an integral part of human society and culture, understanding the mechanisms by which we prefer different types of music may also shed light on the aspects of human society and experience that correspond to these differences.

Our paper also demonstrates the potential uses of machine learning and other computer science methods in cross-cultural research. Given the advent of digital and online media, these repositories of cultural products may hold valuable insight into the diversity of humanity. Computer science as a field has utilised these kinds of data to great effect, be it in developing recommendation systems, or in predicting consumer behaviour, and we hope to demonstrate that these same data and methods can also contribute towards research on society and culture. While a common argument has been that machine learning emphasises prediction, whereas social scientific research prefers interpretation, we show that the two goals are not mutually exclusive. As demonstrated through the use of model interpretation techniques like RFIs and PDPs, supplementing commonly-used prediction focused models in computer science with explanation and model interpretation techniques enables big data and machine learning to offer an efficient and viable means for the empirical analysis of sociocultural phenomenon. At the same time, these methods are more objective, and hold less bias than commonly used methods like selfreports. Particularly for cross-cultural research, future directions could also apply this methodology to identify new avenues of cultural differences in other mediums, as an additional analysis tool. Additionally, computer scientists and engineers could also benefit from this knowledge, as such analyses of sociocultural phenomenon could also aid with the fine-tuning of weights in more opaque deep learning models, such as in recommendation systems used by streaming companies when targeting users from different cultures.

Peer] Comput. Sci. reviewing PDF | (CS-2021:03:58632:2:0:NEW 7 Jun 2021) 
400

401

402

403

404

405

406

407

408

409

410

411

412

413

414

415

416

417

418

419

420

421

422

423

424

425

426

427

428

429

430

431

432

433

434

435

436

437

438

\section{References}

Anderson, C. (2014). The use of traditional Japanese music as an inspiration for modern saxophone compositions: An interpretive guide to Joji Yuasa's Not I but the wind' and Masakazu Natsuda's West, or Evening Song in Autumn [doctoral dissertation]. http://hdl.handle.net/2142/49546, 2014.

Askin, A., Mauskapf, M. (2017). What makes popular cultural popular? Product features and optimal differentiation in music. American Sociological Review, 82(5), 910-944. https://doi.org/10.1177/0003122417728662

Bardi. A., Calogero, R. M., \& Mullen, B. (2008). A new archival approach to the study of values and value--Behavior relations: Validation of the value lexicon. Journal of Applied Psychology, 93(3), 483-497. https://doi.org/10.1037/0021-9010.93.3.483

Bennett, A. (1999). Hip hop am Main: the localization of rap music and hip hop culture. Media, Culture \& Society, 21(1), f 77-91, 1999.

Cheung, F. M. (2012). Mainstreaming culture in psychology. American Psychologist, 67(8), 721730. https://doi.org/10.1037/a0029876.

de Almeida, I., \& Uchida, Y. (2018). Examining affective valence in Japanese and Brazilian cultural products: An analysis on emotional words in song lyrics and news articles. Psychologia, 61(3), 174-184. https://doi.org/10.2117/psysoc.2019-A103.

Deva, D. (1999). Underlying socio-cultural aspects and aesthetic principles that determine musical theory and practice in the musical traditions of China and Japan. Renaissance Artists and Writers Assocation.

Dunbar, R. I. M. (2012). On the evolutionary function of song and dance. In N. Bannan (Ed.), Music, language and human evolution (pp. 201-214). Oxford, England: Oxford University Press.

Febirautami, L. R., Surjandari, I., \& Laoh, E. (2019). Determining characteristics of popular local songs in Indonesia's music market. In Proceedings of the 5th International Conference on Information Science and Control Engineering, Zhengzhou, China.

Fisher, A., Rudin, C., \& Dominici, F. All models are wrong, but many are useful: Learning a variable's importance by studying an entire class of prediction models simultaneously. Journal of Machine Learning Research, 20(177), 1-81.

Friedman, J. H. (2001). Greedy function approximation: A gradient boosting machine, The Annals of Statistics, 29(5), 1189-1232.

Fricke, K. R., Greenberg, D. M., Rentfrow, P. J., \& Herzberg, P. Y. (2019). Measuring musical preferences from listening behavior: Data from one million people and 200,000 songs. Psychology of Music. https://doi.org/10.1177/0305735619868280

Golder, S., \& Macy, M. W. (2011). Diurnal and seasonal mood vary with work, sleep, and daylength across diverse cultures. Science, 333(6051), 1878-1881. https://doi.org/10.1126/science.1202775 
439

440

441

442

443

444

445

446

447

448

449

450

451

452

453

454

455

456

457

458

459

460

461

462

463

464

465

466

467

468

469

470

471

472

473

474

475

476

477

478

Greenwell, B. M. (2017). pdp: An R Package for constructing partial dependence plots. The $R$ Journal, 9(1), 421-436. https://journal.r-project.org/archive/2017/RJ-2017016/index.html.

Greenwell, B., Boehmke, B., Cunningham, J., \& GBM Developers, gbm: Generalized Boosted Regression models. R package version 2.1.5. https://CRAN.R-project.org/package=gbm

Groarke, J. M., \& Hogan, M. J. (2018). Development and psychometric evaluation of the Adaptive Functions of Music Listening Scale. Frontiers in Psychology, 9, 516. https://doi.org/10.3389/fpsyg.2018.00516

Hakvoort, L. (2015). Rap music therapy in forensic psychiatry: Emphasis on the musical approach to rap. Music Therapy Perspectives, 33(2), 184-192. https://doi.org/10.1093/mtp/miv003.

Heine, S. J., \& Hamamura, T. (2007). In search of East Asian self-enhancement. Personality and Social Psychology Review, 11, 4-27. https://doi.org/10.1177/1088868306294587.

Heine, S., \& Ruby, M. B. (2010). Cultural psychology. Wiley Interdisciplinary Reviews, 1(2), 254-266. https://doi.org/10.1002/wcs.7.

Henrich, J., Heine, S., \& Norenzayan, A. (2010). Most people are not WEIRD. Nature, 466, 29. https://doi.org/10.1038/466029a.

Hirst, D. (2013). Melody metrics for prosodic typology: comparing English, French and Chinese. In Interspeech. Annual Conference of the International Speech Communication Association., Lyon, France.

Howlin, C., \& Rooney, B. (2020). Patients choose music with high energy, danceability, and lyrics in analgesic music listening interventions. Psychology of Music. https://doi.org/10.1177/0305735620907155.

Joshanloo M., \& Weijers, D. (2014). Aversion to happiness across cultures: A review of where and why people are averse to happiness. Journal of Happiness Studies, 15, 717-735. https://doi.org/10.1007/s10902-013-9489-9.

Kuhn, M. (2019). caret: Classification and regression training. R package version 6.0-82. https://CRAN.R-project.org/package=caret

Lamoreaux, M., \& Morling, B. (2012). Outside the head and outside individualism-collectivism: Further meta-analyses of cultural products. Journal of Cross-Cultural Psychology, 43(2), 299-327.

Lartillot, O., Toiviainen, P., \& Eerola, T. (2008). A Matlab toolbox for music information retrieval. In C. Preisach, H. Burkhardt, L. Schmidt-Thieme, \& R. Decker (Eds.), Data analysis, machine learning, and applications: Studies in classification, data analysis, and knowledge organization (pp. 261-268). Berlin, Germany: Springer.

Markus, H. R., \& Kitayama, S. (1991). Culture and the self: Implications for cognition, emotion, and motivation. Psychological Review, 98(2), 224-253.

Markus, H. R., \& Kitayama, S. (2010). Cultures and selves: A cycle of mutual constitution. Perspectives on Psychological Science, 5(4), 420-430. https://doi.org/10.1177/1745691610375557. 
479

480

481

482

483

484

485

486

487

488

489

490

491

492

493

494

495

496

497

498

499

500

501

502

503

504

505

506

507

508

509

510

511

512

513

514

515

516

517

518

Mauch, M., MacCallum, R. M., Levy, M., \& Leroi, A. M. (2015). The evolution of popular music: USA 1960-2010. Royal Society Open Science, 2(5), 150081. https://doi.org/10.1098/rsos.150081

Molnar, C. (2019). Interpretable Machine Learning. A Guide for Making Black Box Models Explainable. https://christophm.github.io/interpretable-ml-book/.

Molnar, C., Bischl, B., \& Casalicchio, G. (2019). iml: An R package for interpretable machine learning. Journal of Open Statistical Software, 3(26), 786. doi: 10.21105/joss.00786.

Morling, B. \& Lamoreaux, M. (2008). Measuring culture outside the head: A meta-analysis of individualism-collectivism in cultural products. Personality and Social Psychology Review, 12, 199-221. https://doi.org/10.1177/1088868308318260.

Muthukrishna, M., Bell, A. V., Henrich, J., Curtin, C. M., Gedranovich, A., McInerney, J., \& Thue, B. (2020). Beyond Western, Educated, Industrial, Rich, and Democratic (WEIRD) psychology: Measuring and mapping scales of cultural and psychological distance. Psychological Science, 31(6), 678-701. https://doi.org/10.1177/0956797620916782.

Okuno, T. (2015). Roots of Cool Japan: From the Japanese raditional Edo culture to Anime and Manga," Kwansei Gakuin University Social Sciences Review, 19, 1-7.

Park, M., Thom, J., Mennicken, S., Cramer, H., \& Macy, M. (2019). Global music streaming data reveal diurnal and seasonal patterns of affective preference. Nature Human Behaviour, 3, 230-236. https://doi.org/10.1038/s41562-018-0508-Z.

Pérez-Verdejo, J. M., Piña-García, C. A., Ojeda, M. M., Rivera-Lara, A., \& Méndez-Morales L. (2020). The rhythm of Mexico: an exploratory data analysis of Spotify's top 50. Journal of Computation Social Science. https://doi.org/10.1007/s42001-020-00070-z.

R Core Team, (2019). $R$ : A language and environment for statistical computing. R Foundation for Statistical Computing: Vienna, Austria.

Savage, P. E., Brown, S., Sakai, E., \& Currie, T. E. (2015). Statistical universals reveal the structures and functions of human music. Proceedings of the National Academy of Sciences, 112 (29), 8987-8992. https://doi.org/10.1073/pnas.1414495112.

Schwind, A., Haberzettl, L., Wamser, F., \& Hoßfeld, T. (2019). QoE analysis of Spotify audio streaming and app browsing. In Internet-QoE'19: Proceedings of the 4th Internet-QoE Workshop on QoE-based Analysis and Management of Data Communication Networks.

Smith, P. B., Fischer, R., Vignoles, V. L., \& Bond, M. H. (2013). Understanding social psychology across cultures: Engaging with others in a changing world. Sage.

Thompson, C., Parry, J., \& Wolff, T. (2019). spotifyr: $R$ wrapper for the 'Spotify' web API, R package version 2.1.1. http://github.com/charlie86/spotifyr.

Triandis, H., Marin, G., Lisansky, J., \& Betancourt, H. (1984). Simpatía as a cultural script of Hispanics. Journal of Personality and Social Psychology, 47, 1363-1375.

Tsai, J. L. (2007). Ideal affect: Cultural causes and behavioral consequences. Perspectives on Psychological Science, 2(3), 242-259. https://doi.org/10.1111/j.1745-6916.2007.00043.x.

Uchida, Y., \& Kitayama, S. (2009). Happiness and unhappiness in East and West: Themes and variations. Emotion, 9(4), 441-456. https://doi.org/10.1037/a0015634.

Peer] Comput. Sci. reviewing PDF | (CS-2021:03:58632:2:0:NEW 7 Jun 2021) 
519 Venables, W. N., \& Ripley, B. D. (2002). Modern Applied Statistics with S, New York: 520 Springer. 


\section{Table $\mathbf{1}$ (on next page)}

A list of song-level audio features obtained from Spotify for our analyses

Audio features refer to the music features as listed on the Spotify API, followed by a brief description of each feature. More information on the features are available at: https://developer.spotify.com/documentation/web-api/reference/tracks/get-audio-features/ 
Table 1:

A list of song-level audio features obtained from Spotify for our analyses

\begin{tabular}{|c|c|}
\hline Audio feature & Description \\
\hline Duration & The duration of the music in milliseconds (ms). \\
\hline Mode & If the melody of a track is in a major or minor key. \\
\hline Acousticness & A confidence measure on whether a song is acoustic. \\
\hline Danceability & $\begin{array}{l}\text { The suitability of a song for dancing. This is based on several } \\
\text { musical features, such as tempo, rhythmic stability, regularity, and } \\
\text { beat strength. }\end{array}$ \\
\hline Energy & $\begin{array}{l}\text { A measure of the intensity and activity of a song as perceptually } \\
\text { lound, fast, or noisy. This is based on several musical and spectral } \\
\text { features, such as dynamic range, loudness, timbre, onset rate, and } \\
\text { entropy. }\end{array}$ \\
\hline Instrumentalness & A confidence measure of whether a song contains no vocals. \\
\hline Liveness & $\begin{array}{l}\text { A confidence measure on the presence of audiences in the } \\
\text { recording. }\end{array}$ \\
\hline Loudness & The overall intensity of the song in decibels (dBFS). \\
\hline Speechiness & A confidence measure on the presence of spoken words in a song. \\
\hline Valence & An estimate of whether a song conveys positive or negative affect. \\
\hline Tempo & The estimated main tempo of a song. \\
\hline
\end{tabular}




\section{Table 2 (on next page)}

Medians (L/U quantiles) and missing data for musical features (excluding mode), and release year 
Table 2:

Medians (L/U quantiles) and missing data for musical features (excluding mode), and release year.

\begin{tabular}{|c|c|c|c|c|c|c|}
\hline \multirow[t]{2}{*}{ Feature } & \multicolumn{2}{|c|}{ Chinese } & \multicolumn{2}{|c|}{ Japanese } & \multicolumn{2}{|c|}{ Western } \\
\hline & Median (L/U) & Missing & Median (L/U) & Missing & Median (L/U) & Missing \\
\hline Danceability & $0.56(0.46 / 0.66)$ & 3 & $0.56(0.45 / 0.67)$ & 4 & $0.66(0.55 / 0.76)$ & 1 \\
\hline Energy & $0.46(0.34 / 0.63)$ & 3 & $0.76(0.51 / 0.90)$ & 4 & $0.69(0.54 / 0.82)$ & 0 \\
\hline Loudness & $-8.9(-11.1 /-6.9)$ & 3 & $-6.2(-9.3 /-4.3)$ & 4 & $-6.8(-8.9 /-5.2)$ & 0 \\
\hline Speechiness & $0.04(0.03 / 0.05)$ & 3 & $0.05(0.04 / 0.09)$ & 4 & $0.08(0.04 / 0.24)$ & 0 \\
\hline Acousticness & $0.54(0.21 / 0.76)$ & 3 & $0.11(0.01 / 0.49)$ & 4 & $0.09(0.02 / 0.29)$ & 1 \\
\hline Instrumentalness & $\begin{array}{l}1.4 \mathrm{E}-6(0.00 / 1.1 \mathrm{E}- \\
4)\end{array}$ & 3 & $1.0 \mathrm{E}-4(0.00 / 0.36)$ & 4 & $0.00(0.00 / 0.0006)$ & 1 \\
\hline Liveness & $0.13(0.10 / 0.21)$ & 3 & $0.14(0.10 / 0.29)$ & 4 & $0.14(0.10 / 0.29)$ & 1 \\
\hline Valence & $0.37(0.24 / 0.57)$ & 3 & $0.52(0.31,0.71)$ & 4 & $0.51(0.33 / 0.69)$ & 1 \\
\hline Tempo & $\begin{array}{l}122.7 \\
(100.0 / 138.1)\end{array}$ & 3 & $123.7(99.0 / 144.0)$ & 4 & $119.7(96.6 .136 .2)$ & 0 \\
\hline Duration (ms) & $\begin{array}{l}240213 \\
(205586 / 272586)\end{array}$ & 3 & $\begin{array}{l}236840 \\
(187266 / 281573)\end{array}$ & 4 & $\begin{array}{l}215640 \\
(184727 / 250693)\end{array}$ & 0 \\
\hline Release year & $2011(2005 / 2015)$ & 148 & $2015(2010 / 2018)$ & 100 & $2016(2012 / 2018)$ & 0 \\
\hline
\end{tabular}

1 


\section{Table 3(on next page)}

Comparison of feature importance between the GBDT and MLP multiclass models 
Table 3:

Comparison of feature importance measures.

\begin{tabular}{lll}
\hline Feature & RFI (GBDT) & PFI (MLP) \\
\hline Speechiness & 24.3 & 1.26 \\
Loudness & 7.4 & 1.15 \\
Instrumentalness & 15.6 & 1.19 \\
Acousticness & 16.5 & 1.14 \\
Energy & 17.5 & 1.12 \\
Mode name & 0.1 & 1.00 \\
Duration & 8.4 & 1.12 \\
Danceability & 5.9 & 1.06 \\
Valence & 1.8 & 1.03 \\
Tempo & 2.2 & 1.01 \\
Liveness & 0.2 & 1.00 \\
\hline
\end{tabular}

1 


\section{Table 4(on next page)}

Comparison of feature importance measures for respective binomial classification models

Note: While scales between RFI and PFI are not equivalent, both measure model-specific feature importance relative to other features: the higher the score, the larger the importance within the model. Features with highest importance are in bold. PFIs were reported with 2 decimal places, but we used 3 decimal places for PFls denoted by '^’. This was to identify the $2^{\text {nd }}$ most important feature for the PDP. 
Table 4:

Comparison of feature importance measures for respective binomial classification models.

Note: While scales between RFI and PFI are not equivalent, both measure model-specific feature importance relative to other features: the higher the score, the larger the importance within the model. Features with highest importance are in bold. PFIs were reported with 2 decimal places, but we used 3 decimal places for PFIs denoted by '^’ . This was to identify the $2^{\text {nd }}$ most important feature for the PDP.

\begin{tabular}{|c|c|c|c|c|c|c|}
\hline \multirow[t]{2}{*}{ Feature } & \multicolumn{2}{|c|}{ Chinese-Japanese } & \multicolumn{2}{|c|}{ Chinese-English } & \multicolumn{2}{|c|}{ Japanese-English } \\
\hline & $\begin{array}{l}\text { RFI } \\
\text { (GBDT) }\end{array}$ & PFI (MLP) & $\begin{array}{l}\text { RFI } \\
\text { (GBDT) }\end{array}$ & PFI (MLP) & $\begin{array}{l}\text { RFI } \\
\text { (GBDT) }\end{array}$ & PFI (MLP) \\
\hline Speechiness & 4.8 & 1.05 & 46.6 & 1.67 & 39.6 & 1.22 \\
\hline Loudness & 14.1 & 1.46 & 3.0 & 1.19 & 4.7 & 1.04 \\
\hline Instrumentalness & 26.0 & 1.22 & 2.8 & 1.08 & 8.5 & $1.105^{\wedge}$ \\
\hline Acousticness & 31.1 & 1.16 & 34.1 & 1.35 & 2.4 & 1.04 \\
\hline Energy & 32.9 & 1.07 & 4.7 & 1.09 & 16.0 & $1.114^{\wedge}$ \\
\hline Mode name & 0.0 & 1.00 & 0.0 & 1.01 & 0.2 & 1.00 \\
\hline Duration & 9.3 & 1.08 & 4.3 & 1.07 & 12.9 & 1.02 \\
\hline Danceability & 1.2 & 1.01 & 1.6 & 1.06 & 10.4 & $1.110^{\wedge}$ \\
\hline Valence & 0.7 & 1.06 & 1.2 & 1.02 & 3.3 & 1.003 \\
\hline Tempo & 1.4 & 1.01 & 1.5 & 1.02 & 1.9 & 1.00 \\
\hline Liveness & 0.0 & 1.00 & 0.2 & 1.00 & 0.1 & 1.00 \\
\hline
\end{tabular}


Figure 1

Number of songs (in our data) by year for Japanese (JP), English (US), and Chinese (ZH) medium songs

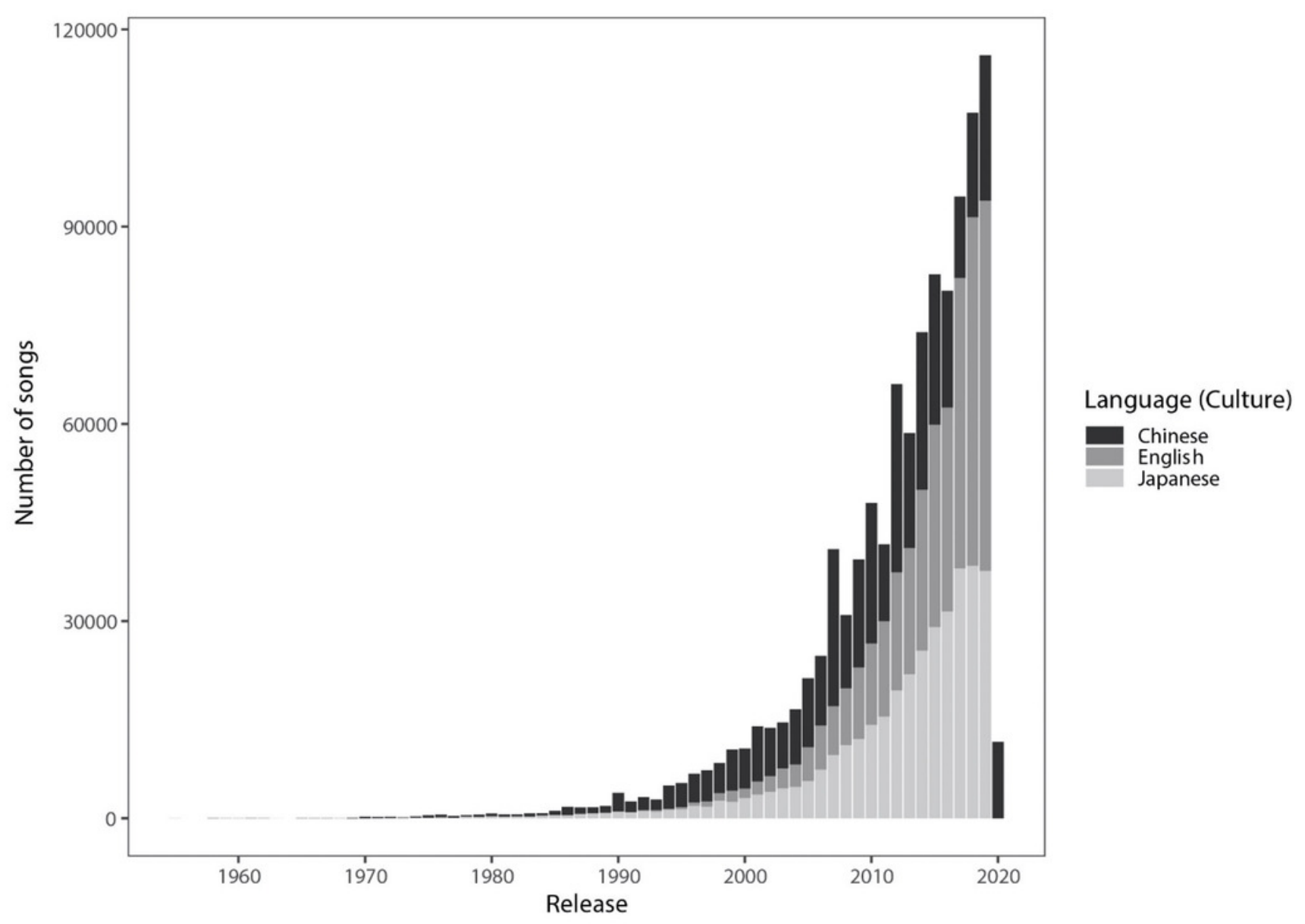

Figure 1: Number of songs (in our data) by year for Japanese (JP), English (US), and Chinese (ZH) medium songs. 
Figure 2

PDPs of top 2 most important features in each model on the probability of classification

The positive class is indicated at the top of the Y-axis. For example, in the Japanese-English GBDT model (top right) for the speechiness feature, the decreasing trend indicates that the higher the speechiness score, the lower the probability of classification (of a song) as being Japanese (i.e., higher probability of being English), in a fairly linear fashion.
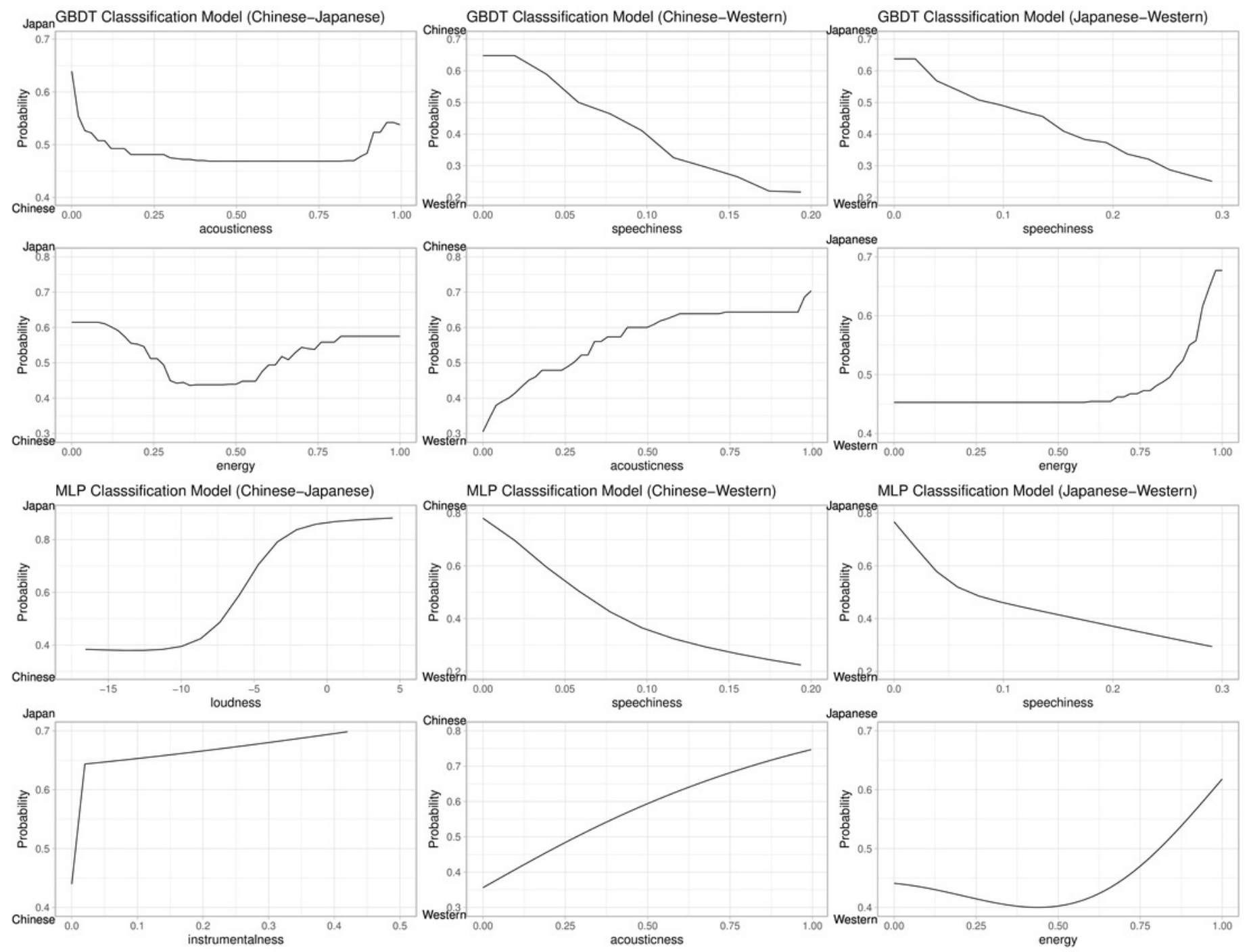
Figure 3

Variation and stability of features (medians) across cultures from 2000 to 2020

Note that scales differ bewteen features.
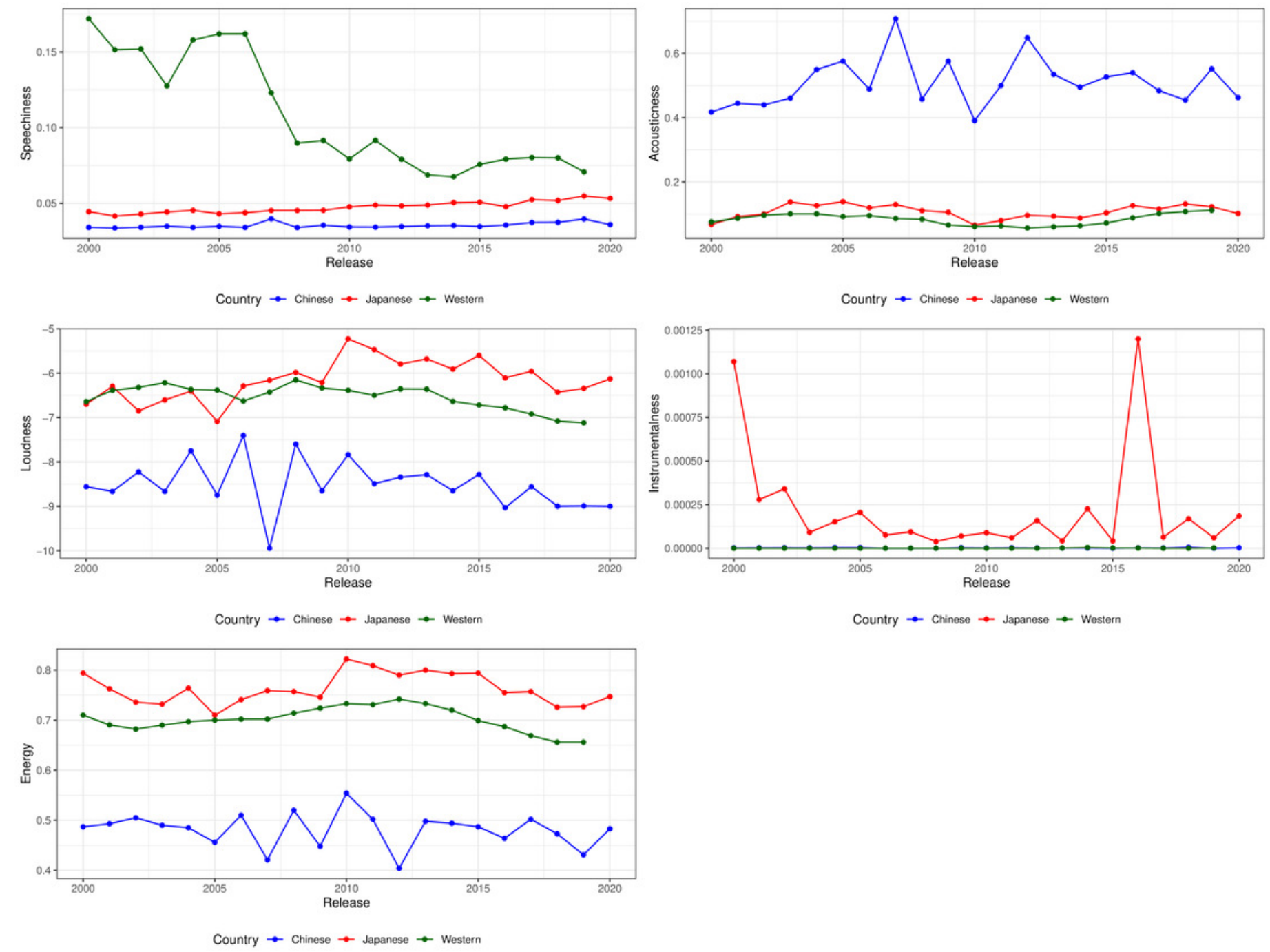

Country $\rightarrow$ Chinese $\rightarrow$ Japanese $\rightarrow$ Western 